\title{
Cystic Variation of Schwannoma in the Neck: A Rare Presentation
}

Riya Ramteke, Swanit H. Deshpande, Roshan Chiranjeev, Jayashri S. Pandya*

MS General Surgery, MBBS, B.Y.L Nair Charitable Hospital.

*Corresponding Author: Jayashri S. Pandya, MS General Surgery, MBBS, B.Y.L Nair Charitable Hospital.

Received date: October 06, 2020; Accepted date: November 09, 2020; Published date: November 13,2020

Citation: R Ramteke, Swanit H. Deshpande, R Chiranjeev, Jayashri S. Pandya. (2020) Cystic Variation of Schwannoma in the Neck: A Rare Presentation. International Journal of Clinical Case Reports and Reviews. 4(3); DOI: 10.31579/2690-4861/076

Copyright: (c) 2020 Jayashri S. Pandya, This is an open-access article distributed under the terms of the Creative Commons Attribution License, which permits unrestricted use, distribution, and reproduction in any medium, provided the original author and source are credited.

\section{Abstract}

It is uncommon for schwannomas to present as neck swellings. This case presented as painless, cystic and slow growing neck swelling which was excised. Histopathology proved it to be cystic schwannoma. Cystic change occurs in only $4 \%$ of all schwannomas. The cystic change renders the schwannoma to mimic second branchial cyst on clinical and radiological examination. Although modality of treatment remains the same which is extracapsular excision, the follow up differs in terms of recurrence.

Keywords: schwannomas; neck swelling; radiological examination

\section{Background}

Common differential diagnoses for benign neck masses are branchial cyst and necrotic cervical lymphadenopathy. Benign cystic lateral masses that can mimic branchial cleft cyst include thyroglossal cyst, carotid body tumours, ectopic thymic cyst, lymphangiomas, dermoid, cystic schwannoma epidermoid cysts and cystic nerve sheath tumours. [1]

Schwannomas are capsulated, slow growing, benign tumours arising from schwann cells of myelinated nerves, commonly called as neurilemmomas. They may arise from any peripheral, cranial or autonomic nerve. More than $25 \%$ Schwannomas are found in head and neck region. [2] Schwannoma of neck is a relatively uncommon tumor. [3] Origin of the schwannoma in the neck is more commonly from vagus and very rarely from cervical sympathetic chain. Cervical vagal Schwannoma constitute $2-5 \%$ of neurogenic tumours.

There are two types of tissues in schwannoma viz Antoni A and Antoni B. The layering with fluid levels is caused by hemorrhages. Usually Antoni B undergoes cystic change and hemorrhagic degeneration. The cystic variants comprise of $4 \%$ tumours. $[1,4,5]$ The cystic Schwannoma grows more rapidly than non-cystic type.

Most common cystic mass in the neck is branchial cleft cyst. Branchial cleft cyst most common congenital cyst arising anterior to sternocleidomastoid.

This case is a rare presentation of cystic schwannoma demonstrating the classic location and CT signs of a branchial cyst in a 36-year female patient with left sided neck mass. Tumour was excised and histopathology revealed the tumour to be cystic schwannoma.

\section{Case Presentation}

Thirty six years old female presented with solitary painless cystic swelling over left side of the neck, which was insidious in onset and gradually increasing in size over the past five years. The swelling caused no change in voice, neurological deficit like numbness, pain, dysphagia, weakness or facial nerve palsy.
Patient has history of pulmonary tuberculosis in her childhood for which she took complete treatment. Patient had no other co-morbidities.

On local examination, there was a left sided $4 * 3 \mathrm{~cm}$ single cystic fusiform swelling arising from lower $1 / 3 \mathrm{rd}$ of anterior triangle of neck at the level of cricoid, crossing middle 3rd of sternocleidomastoid muscle and reaching postero-superior boundary of posterior triangle of neck. It was $1 \mathrm{~cm}$ below left mastoid tip.

Swelling was non tender, non-pulsatile, slightly mobile and did not move with deglutition. Bilateral carotid pulsations were felt. The skin over swelling was freely mobile and was not associated with any skin changes. There was no paresthesia over skin. [Figure 1]

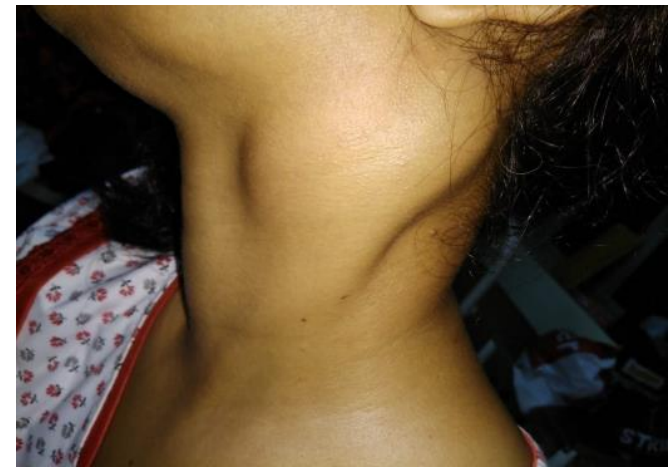

Figure 1: depicts the clinical photograph depicting the swelling in a image captured from a left lateral position while the patient was in an erect posture.

Oral examination showed normal tonsillar fossa. There was no evidence of internal opening or sinus tract. No obvious bulge or displacement of peritonsillar structures was seen. Uvula was central in position. Gag reflex was present.

70 degree scopy revealed bilateral vocal cords free and mobile. Parapharyngeal space examination was normal. 


\section{Investigations}

Ultrasound neck report revealed a well circumscribed hypoechoic soft tissue lesion with internal vascularity along antero-lateral aspect of internal and external carotid arteries and closely abutting internal carotid artery. USG Doppler of neck revealed fairly well defined, oval, anechoic cystic lesion with smooth walls measuring $55 * 47 * 30 \mathrm{~mm}$. The lesion had multiple internal septations. The lesion was located in the left carotid space anterior to left sternocleidomastoid. It was suspected to be compressing superior $1 / 3$ rd of internal jugular vein (IJV) posteriorly. Distal and mid 1/3rd of IJV showed normal course, caliber and colour flow.

CT scan showed a well differentiated multi-septate predominantly cystic lesion with a few enhancing solid components at periphery of approx. $65 * 42 * 30 \mathrm{~mm}$ in left carotid space. No evidence of any lymphadenopathy. The lesion was located in the left carotid space anterior to left sternocleidomastoid.

Fine needle aspiration cytology (FNAC) of the lesion showed benign cystic lesion and was inconclusive.

\section{Differential Diagnosis}

On clinical history, examination and investigations, the primary diagnosis was branchial cyst from 2 nd cleft with differential of cystic Schwannoma.

\section{Treatment}

Decision was taken to surgically remove the tumour. Extracapsular enucleation of tumour was done. Curvilinear skin incision was taken. A cystic swelling $3.3 * 2.1 * 0.9 \mathrm{~cm}$ in subplatysmal plane was visualized. The tumour had irregular surface with extension behind sternocleidomastoid. It was abutting the adjacent soft tissues and blood vessels. The cystic tumour was separated and extracapsular enucleation was performed. Swelling was related anteriorly to sternocleidomastoid, medially to internal jugular vein and vagus nerve, supero-posteriorly to marginal mandibular nerve. It was found arising from vagus nerve.

Marginal mandibular nerve was flush to swelling in its superior-posterior aspect.

On microscopic examination the tumour was well circumscribed. It showed large cystic areas and cystic spaces, lined by spindle tumour cells which were arranged in bundle in streaming fashion. Individual cells had moderate eosinophilic cytoplasm with elongated wavy nuclei which at places showed Varocay body formation. Some of these foci showed increased intervening matrix, representing Antony B areas. Some areas showed crowding of cells representing Antony A areas. There were well distributed dilated and congested spaces. Vascular channels were seen. No mitoses or areas of necrosis were identified. [Fig 2,3] These findings confirmed the diagnosis of schwannoma with cystic change.

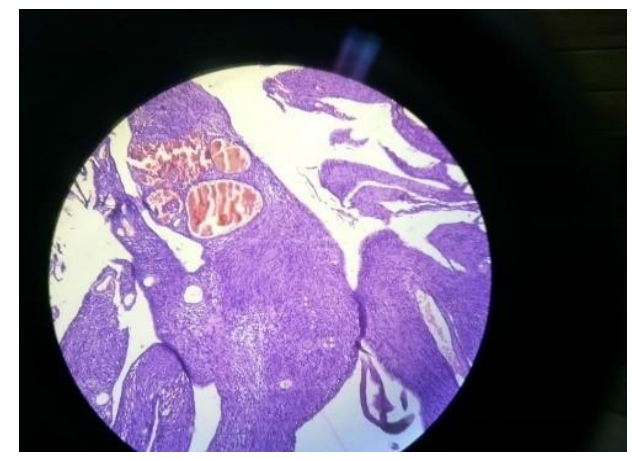

Figure 2: Shows a well circumscribed tumour. It showed large cystic areas and cystic spaces, lined by spindle tumour cells which were arranged in bundle in streaming fashion.

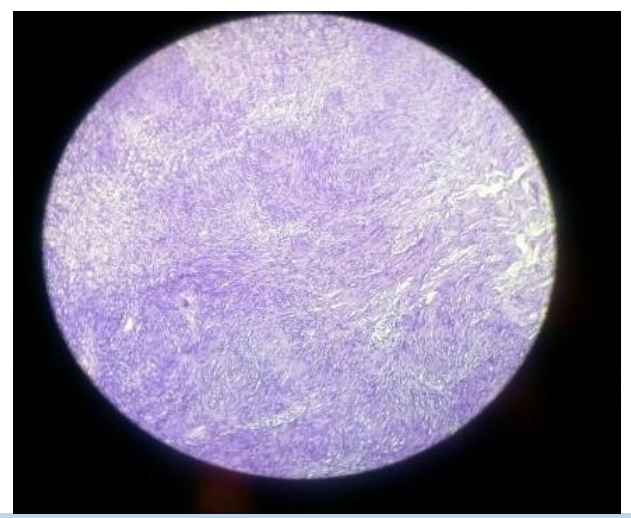

Figure 3: High power image showing foci of increased intervening matrix, representing Antony B areas and areas showing crowding of cells representing Antony a areas.

\section{Outcome and Follow-Up}

The histopathology confirmed diagnosis of Cystic Schwannoma arising from Vagus nerve.

Patient had minimum post-operative deviation of angle of mouth, mild ptosis of left eyelid, marginal mandibular nerve paresis and features suggestive of First bite syndrome. Patient recovered completely with physiotherapy in two weeks after surgery.

There was no vocal cord paralysis. Patient was asymptomatic after 2 months follow up.

\section{Discussion}

The clinical and radiological examination by virtue of the location of the tumour makes branchial cyst as the first differential diagnosis. Schwannomas rarely recur. In cases of branchial cyst, a long term followup is necessary as it tends to recur.

Varocay first reported Schwannoma in 1910. Schwannnomas are nerve sheath tumours which arise from cells of neural sheath. They may arise from any central, peripheral or autonomic nerve. Schawannoma can arise from all cranial nerves, most common being 8th cranial nerve.[2] However in approximately $10-40 \%$ cases origin of nerve remains unidentified. The most common location of schwannomas of head and neck is parapharyngeal space. Schwannoma of neck is a relatively uncommon tumour. Cervical vagal schwannoma constitutes $2-5 \%$ of neurogenic tumours. Other locations include submandibular space, paranasal sinuses, cheek and oral cavity which are rare.

When arising from parapharyngeal space, they present as asymptomatic, slow growing neck masses. Pre- operative diagnosis is difficult as most patients do not have neurological deficit. Hence, other common neck swellings may be considered as differential diagnoses ahead of schwannomas. More common neck swellings are branchial cyst, paraganglioma, lymphoma and metastatic cervical lymphadenopathy.

Schwannomas often present as solitary, painless, slow growing mass of variable size. Cystic Schwannoma grows more rapidly than non-cystic Schwannoma. Hoarseness is the most common presenting symptom. Elicitation of paroxysmal cough on palpation of mass is a specific but rare clinical sign seen in vagal Schwannomas.

The diagnostic difficulty may be further compounded by marked cystic changes which is present in only $4 \%$ of cases. The cystic change is attributed to necrosis, mucinous degeneration, and hemorrhage and microcyst formation.

The first documented case of a cystic Schwannoma of neck masquerading as branchial cyst clinically and on ultrasonography was reported by Buchanan et al. [4] 
In our patient on clinical examination, it was difficult to differentiate between branchial cyst and cystic schwannoma as both can present with painless slow growing mass without any symptoms in lateral neck. [2, 5] On Ultrasound examination, Branchial Cyst shows variable echogenicity but no internal vascularity. Schwannomas on colour doppler show internal vascularity. [6] In our patient the ultrasound did document internal vascularity.

Fine Needle Aspiration Cytology (FNAC) was inconclusive. Usually in Schwannoma due to hemorrhagic aspirate or inadequate cellular material Decision for surgical excision was taken and extracapsular enucleation was done. It was found intraoperatively arising from vagus.

Learning Points/Take Home Messages

- Although Branchial Cyst is more common but while dealing with cystic swelling of neck, the possibility of schwannoma should also be considered.

- Cystic schwannoma can present as branchial cyst radiologically and clinically but meticulous surgical dissection, tracing the site of origin of the mass and final histopathology report are guides towards correct diagnosis.

- It is important to look for signs and symptoms of neurological deficit in the post-operative period.

- Complete surgical excision is treatment of choice in symptomatic cystic schwannomas.

\section{References}

1. Gupta S,Kumar V, Verma R. (2016) Schwannoma mimicking branchial cleft cyst- A case Report.J Adv Med Dent Scie Res. 4(5):75-77.

2. Sharma DK, Sohal BS, Parmar TL, Arora H. (2012) Schwannomas of head and neck and review of literature. Indian J Otolaryngol Head Neck Surg. 64:177-180.

3. Buchanan MA,Williams SM, Hellquist H, Innes AJ. (2009) Cystic schwannoma of cervical plexus masquerading as type 2 second branchial cleft cyst. Eur Arch Otorhinolaryngo. 266:459-462.

4. Bohara S, Dey B, Agarwal S, Gupta R, Khurana N, Gulati A. (2014) A case of Cystic Schwannoma in neck masquerading as Branchial Cleft Cyst. PMC. 6(3):53-55.

5. Wakoh M, Yonezu H, Otonari T, et al. (2005) Two cases of Schwannoma with marked cystic changes. Dentomaxillofac radiol.34; 44-50.

6. Gritzmann N,Hollerweger A, Macheiner P, Rettenbacher $\mathrm{T}$. (2002) Sonography of soft tissue masses of the neck. J Clin Ultrasound.30:356-373. 